Article

\title{
Analysis of Magneto-Mechanical Response for Magnetization-Graded Ferromagnetic Material in Magnetoelectric Laminate
}

\author{
Hai Zhou ${ }^{1}$, Feihu Yu ${ }^{1}$, Xueling Jiang ${ }^{1}$, Caijiang Lu ${ }^{1, *}$, Zhongqing Cao ${ }^{1}$, Xiang Chen ${ }^{1}$, \\ Hongli Gao ${ }^{1}$ and Aichao Yang ${ }^{2}$ \\ 1 Department of Electromechanical Measuring and Controlling, School of Mechanical Engineering, \\ Southwest Jiaotong University, Chengdu 610031, China; zhouhai131@163.com (H.Z.); \\ yfhdmail@163.com (F.Y.); 18384278391@163.com (X.J.); zqcao@swjtu.edu.cn (Z.C.); \\ chenxiang_189@163.com (X.C.); hongli_gao@swjtu.edu.cn (H.G.) \\ 2 Jiangxi Electric Power Research Institute, Nanchang 330096, China; dkyyac2015@163.com \\ * Correspondence: lucaijiang@swjtu.edu.cn or lucjpaper@163.com
}

Received: 25 March 2020; Accepted: 15 June 2020; Published: 22 June 2020

check for updates

\begin{abstract}
This paper analyzes the dynamic magneto-mechanical response in magnetization-graded ferromagnetic materials (MGFM) comprised of high-permeability Finemet and traditional magnetostrictive materials. The theoretical modeling of the piezomagnetic coefficient that depends on the bias magnetic field of MGFM is proposed by using the nonlinear constitutive model of a piezomagnetic material, the magnetoelectric equivalent circuit method, and the simulation software Ansoft. The theoretical variation of piezomagnetic coefficients of MGFM on the bias magnetic field is in good agreement with the experiment. Using the piezomagnetic coefficient in the magnetoelectric voltage model, the theoretical longitudinal resonant magnetoelectric voltage coefficients have also been calculated, which are consistent with the experimental values. This theoretical analysis is beneficial to comprehensively understand the self-biased piezomagnetic response of MGFM, and to design magnetoelectric devices with MGFM.
\end{abstract}

Keywords: piezomagnetic coefficient; magnetization-graded ferromagnetic material; magnetoelectric device

\section{Introduction}

Magnetoelectric (ME) laminate composites comprising of piezomagnetic and piezoelectric phases have been extensively investigated in recent years for use in potential smart devices due to their low cost, lightweight, and flexible features [1-3]. The principal of ME response can be determined from the product properties of the piezomagnetic and piezoelectric phases. The ME coefficient is expressed as

$$
\alpha_{M E}=k_{c} \times d_{m} \times d_{p}
$$

where $k_{c}$ is a coupling factor $\left(0 \leq k_{c} \leq 1\right)$ between the two phases, $d_{m}$ is the piezomagnetic coefficient, and $d_{p}$ is the piezoelectric coefficient. The $d_{m}$ and $d_{p}$ are decided by the choice of piezomagnetic materials (Terfenol-D, Metglas, Gafenol, etc.) and piezoelectric materials $\left(\mathrm{Pb}\left(\mathrm{Zr}_{1-\mathrm{x}} \mathrm{Ti}_{\mathrm{x}}\right) \mathrm{O}_{3}\right.$ ceramics, PMN-PT single crystal, AlN films, etc.), respectively [1-3]. The coupling factor $k_{c}$, according to previous reports, is mainly influenced by configuration [4-6], boundary conditions [7], and composite mode (epoxy bonding [8], magnetron sputtering [9], and spin-wave interactions [10-12]). The configuration and boundary conditions determine the mechanical structure of ME composites. The composite mode determines the interface coupling between two phases. 
For most magnetostrictive materials with a small magnetic hysteresis, the value of $d_{m}$ is near zero without an external magnetic field [1-9]. Thus, a magnetoelectric composite requires a DC biased magnetic field $\left(H_{\mathrm{dc}}\right)$ to obtain an enhanced value of $d_{m}$ [13]. Generally, the $H_{\mathrm{dc}}$ is provided by a pair of permanent magnets resulting in a possible noise source in the sensor array, a reduction of the spatial resolution, and an increase in the device volume.

For weakening the dependence of $H_{\mathrm{dc}}$, the self-biased piezomagnetic effect has been presented by researchers. Using the inherent remanence of magnetic material is an effective way [14-16], but the self-biased ME coupling is still weak. The antiferromagnetic-ferromagnetic exchange coupling effect is the other effective method $[17,18]$, but the processing technology is complex. Beyond that, the ME laminate with magnetization-graded ferromagnetic materials (MGFM) consisting of high-permeability ribbons and traditional magnetostrictive materials also has an effective self-biased ME response [19-23]. Up until now, the experimental self-biased ME response for the asymmetric piezoelectric/MGFM laminate and the symmetric MGFM/piezoelectric/MGFM structure have been studied [19-23]. However, up until now, few studies have focused on the theoretical magneto-mechanical response for MGFM.

In this paper, we analyze the magneto-mechanical response of MGFM consisting of Finemet and traditional piezomagnetic layers ( $\mathrm{Ni}$ or FeNi alloy). The theoretical modeling of the magneto-mechanical behavior of MGFM is established using the nonlinear piezomagnetic constitutive model and the method of the equivalent circuit. Compared with the experimental data, the bias field dependences of piezomagnetic coefficients are in good agreement with the calculation from the presented model.

\section{Theoretical Analysis}

\subsection{Dynamic Effective Piezomagnetic Coefficient $d_{33, m}$ of MGFM}

The MGFM FeCuNbSiB/Ni is shown in Figure 1. The phase $m_{1}$ is Finemet (FeCuNbSiB), phase $m_{2}$ is traditional magnetostrictive material (Ni or FeNi). According to the principle of wave mechanics, the displacement direction of the laminate is the same as the propagation direction of the vibration, so the wave formed by elastic vibration is a longitudinal wave. Under the free boundary conditions, the MGFM vibrates freely.

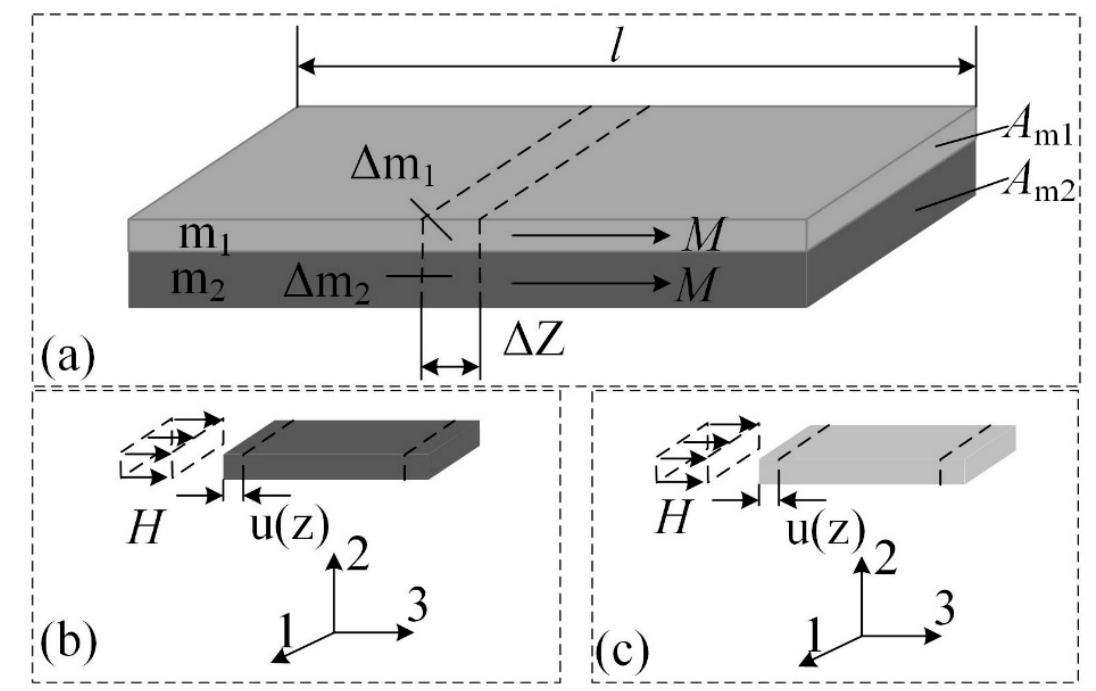

Figure 1. (a) Schematic diagram of magnetization-graded ferromagnetic material $\mathrm{FeCuNbSiB} / \mathrm{Ni}$. The local coordinate systems in (b) FeCuNbSiB ribbon and (c) Ni plate when the magnetic field is applied along the longitudinal 3-direction. The symbol $M$ is magnetization. The $m_{1}$ and $m_{2}$ is the mass of the FeCuNbSiB ribbon and Ni plate, respectively. The $\Delta m_{1}$ and $\Delta m_{2}$ are small mass units in the FeCuNbSiB ribbon and Ni plate, respectively. The $A_{m 1}$ and $A_{m 2}$ is the cross-sectional area of the $\mathrm{FeCuNbSiB}$ ribbon and Ni plate, respectively. The $l$ is the length of $\mathrm{FeCuNbSiB} / \mathrm{Ni}$. The $H$ is the applied magnetic field. The $u(z)$ is the displacement. 
Then, the mechanical vibration equation can be derived as follows $[24,25]$

$$
\left\{\begin{array}{l}
F_{1}=Z_{1} \dot{u}_{1}+Z_{2}\left(\dot{u}_{1}-\dot{u}_{2}\right)+\left(\varphi_{m 1}+\varphi_{m 2}\right) H_{3} \\
F_{2}=-Z_{1} \dot{u}_{2}+Z_{2}\left(\dot{u}_{1}-\dot{u}_{2}\right)+\left(\varphi_{m 1}+\varphi_{m 2}\right) H_{3}
\end{array}\right.
$$

where $Z_{1}=j \rho v A \tan \frac{k l}{2}, Z_{2}=\frac{\bar{\rho} v A}{j \sin k l}, \varphi_{m 1}=\frac{A_{m 1} d_{33, m 1}}{s_{33, m 1}^{H}}, \varphi_{m 2}=\frac{A_{m 2} d_{33, m 2}}{s_{33, m 2}^{H}}, F_{1}$ and $F_{2}$ are forces at the two end faces of the structure, and the $\dot{u}_{1}$ and $\dot{u}_{2}$ are the corresponding displacements. $\bar{\rho}=\left(\rho_{m 1} t_{m 1}+\rho_{m 2} t_{m 2}\right) / t$ is the average density of the laminate. $\varphi_{m 1}$ and $\varphi_{m 2}$ are the magneto-elastic coupling factor. The $d_{33, m 1}$ and $d_{33, m 2}$ is the longitudinal piezomagnetic coefficient of FeCuNbSiB and Ni, respectively. $\bar{\rho}$ is the average density of the laminate. The $k$ is the wavenumber and $v$ is the sound velocity. $s_{33, m 1}^{H}$ and $s_{33, m 2}^{H}$ is the elastic compliance under constant $H$ for $\mathrm{FeCuNbSiB}$ and $\mathrm{Ni}$, respectively.

In this paper, the method of equivalent circuit is used to deal with the magneto-elastic interaction, as shown in Figure 2.

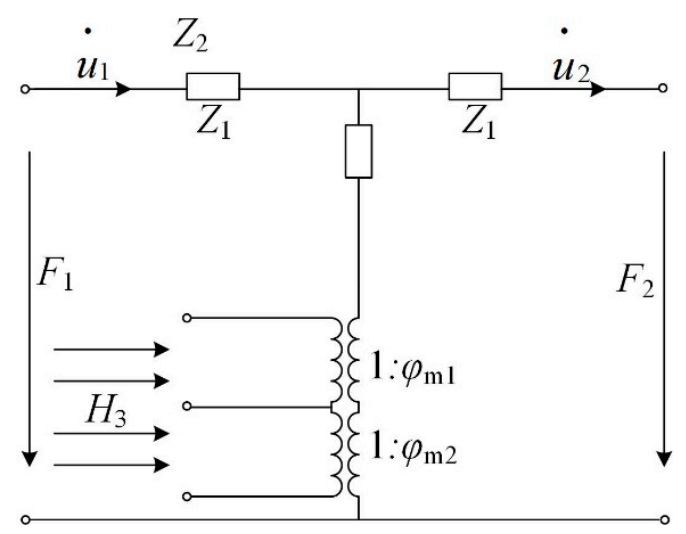

Figure 2. Magneto-elastic equivalent circuit of the magnetization-graded ferromagnetic materials (MGFM).

From the equivalent circuit, the relationship for $\varphi_{m}$ of $\mathrm{FeCuNbSiB} / \mathrm{Ni}, \varphi_{m 1}$ of $\mathrm{FeCuNbSiB}$ phase and $\varphi_{m 2}$ of $\mathrm{Ni}$ phase is

$$
\varphi_{m}=\varphi_{m 1}+\varphi_{m 2}
$$

Then

$$
\frac{\left(A_{m 1}+A_{m 2}\right) d_{33, m}}{\bar{s}}=\frac{A_{m 1} d_{33, m 1}}{s_{33, m 1}^{H}}+\frac{A_{m 2} d_{33, m 2}}{s_{33, m 2}^{H}}
$$

where $\bar{s}$ is the average compliant coefficient and $\frac{1}{\bar{s}}=\frac{t_{m 1}}{\left(t_{m 1}+t_{m 2}\right) s_{33, m 1}^{H}}+\frac{t_{m 2}}{\left(t_{m 1}+t_{m 2}\right) s_{33, m 2}^{H}} \cdot t_{m 1}$ and $t_{m 2}$ are the thicknesses of FeCuNbSiB and Ni, respectively. So, the $d_{33, m}$ of MGFM is

$$
d_{33, m}=\frac{t_{m 1} d_{33, m 1} s_{33, m 2}^{H}+t_{m 2} d_{33, m 2} s_{33, m 1}^{H}}{t_{m 1} s_{33, m 2}^{H}+t_{m 2} s_{33, m 1}^{H}}
$$

\subsection{Effective Dynamic Piezomagnetic Coefficient $d_{33, m 1}$ of FeCuNbSiB in MGFM}

According to the nonlinear magnetostrictive constitutive relationship, the $d_{33, m}$ of magnetostrictive material is [26]

$$
d_{33, m}=\frac{\partial \varepsilon}{\partial H}=\frac{\partial \varepsilon}{\partial M} \frac{\partial M}{\partial H}=2 \lambda_{s}\left(\operatorname{coth}(\eta H)-\frac{1}{\eta H}\right) \times\left[\eta\left(1-\operatorname{coth}(\eta H)^{2}\right)+\frac{1}{\eta H^{2}}\right]
$$


where $M$ is the magnetization, $\varepsilon$ is the stain, $\lambda_{s}$ is the saturation magnetostrictive coefficient and $H$ is the external magnetic field. $\eta=3 \chi_{m} / M_{S} \chi_{m}$ is the initial magnetic susceptibility, $M_{S}$ is the saturation magnetization.

Due to the remanent magnetism of $\mathrm{Ni}$, an additional magnetic field $H_{f}$ in $\mathrm{FeCuNbSiB}$ is generated. Thus, the effective magnetic field of the FeCuNbSiB ribbons in the FeCuNbSiB/Ni laminate $\left(H_{\text {eff, } m 1}\right)$ is

$$
H_{e f f, m 1}=\frac{H_{\mathrm{dc}}+H_{\mathrm{ac}}+H_{f}}{1+N_{d, m 1}\left(\mu_{r, m 1}-1\right)}
$$

where $N_{\mathrm{d}, m 1}$ is the demagnetizing factor of FeCuNbSiB. $H_{\mathrm{dc}}$ is the bias magnetic field. $H_{\mathrm{ac}}$ is the alternating current magnetic field. Therefore, the effective dynamic effective piezomagnetic coefficient $d_{33, m 1}$ of $\mathrm{FeCuNbSiB}$ in the $\mathrm{FeCuNbSiB} / \mathrm{Ni}$ laminate is

$$
d_{33, m 1}=2 \lambda_{s, m 1}\left(\operatorname{coth}\left(\eta_{m 1} H_{e f f, m 1}\right)-\frac{1}{\eta_{m 1} H_{e f f, m 1}}\right) \times\left[\eta_{m 1}\left(1-\operatorname{coth}\left(\eta_{m 1} H_{e f f, m 1}\right)^{2}\right)+\frac{1}{\eta_{m 1}\left(H_{e f f, m 1}\right)^{2}}\right]
$$

In order to obtain the value of $H_{f}$, we use the magnetic field simulation software, Ansoft 11.0. In the simulation, the residual magnetization of $\mathrm{Ni}$ is assumed as the parameter input, so Ni provides the magnetic field $H_{f}$. For magnetostrictive Ni with cubic magnetocrystalline anisotropy and $\left|K_{1}\right|<M_{S}^{2}$, the remanent magnetization is $M_{r}=0.866 M_{s}, \mu_{0} M_{s}=0.616 \mathrm{~T}$. The simulation settings are as follows; $\mathrm{Ni}: \mu_{0} M_{r}=0.534 \mathrm{~T}, \mu_{r}=220$; dimensions are $12 \times 6 \times 1 \mathrm{~mm}^{3} ; \mathrm{FeCuNbSiB}: \mu_{r}=30,000$; dimensions are $12 \times 6 \times 0.120 \mathrm{~mm}^{3}$.

Figure $3 a, b$ illustrates the distributions of magnetic fields of the $\mathrm{FeCuNbSiB} / \mathrm{Ni}$ laminate and the $\mathrm{FeCuNbSiB}$ ribbon in $\mathrm{FeCuNbSiB} / \mathrm{Ni}$, respectively. In the absence of $H_{\mathrm{dc}}$, it can be clearly displayed from Figure 3 that the Ni plate provided a $H_{\mathrm{dc}}$ to $\mathrm{FeCuNbSiB}$ due to the residual magnetization. When the layer of FeCuNbSiB ribbon $L=1, H_{f}=276.78(\mathrm{~A} / \mathrm{m})$.

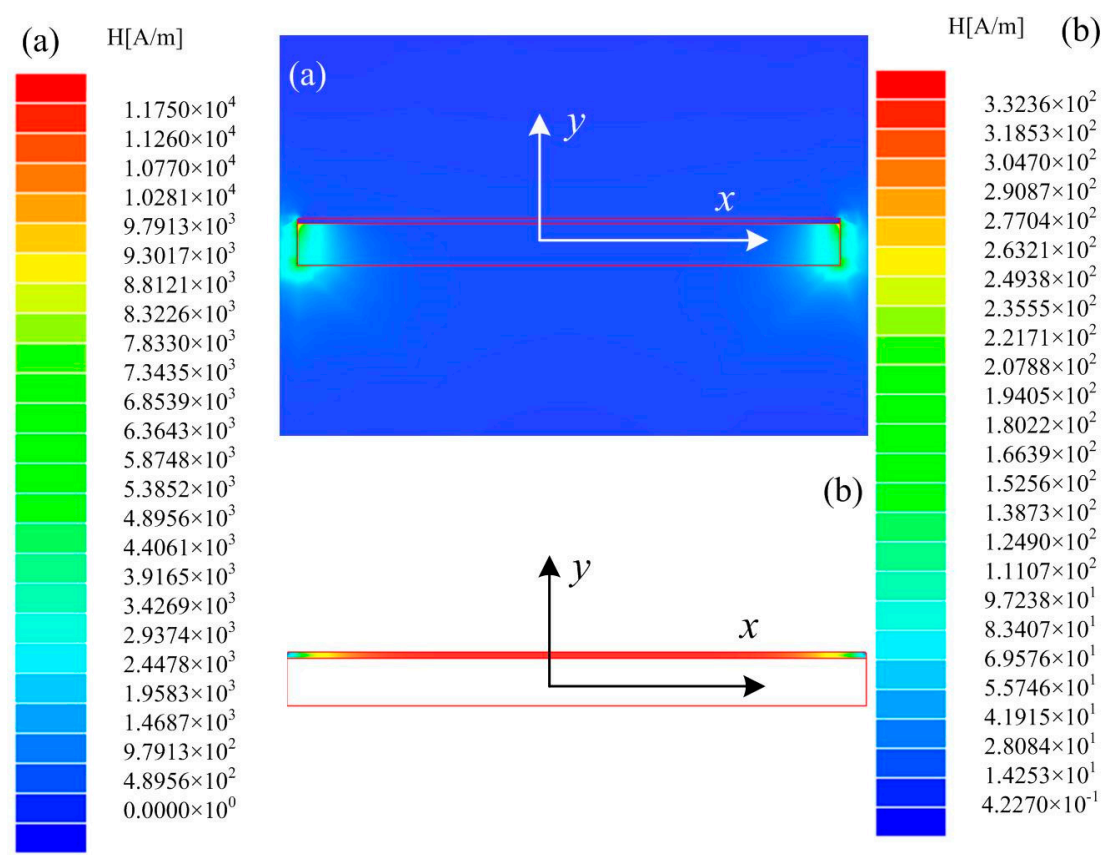

Figure 3. (a) The distribution diagrams of magnetic fields of the FeCuNbSiB/Ni laminate, and (b) The distribution diagrams of magnetic fields of the $\mathrm{FeCuNbSiB}$ ribbon in the $\mathrm{FeCuNbSiB} / \mathrm{Ni}$ laminate.

\subsection{Dynamic Effective Piezomagnetic Coefficient $d_{33, m 2}$ of Ni Plate in MGFM}

For $\mathrm{FeCuNbSiB}$ as a soft magnetic material, the permeability $\mu_{r f}=30,000$, saturation magnetization $\mu_{0} M_{s}=1.45 \mathrm{~T}$, and the anisotropic constant is $-30,000 \mathrm{~J} / \mathrm{m}^{3}$. Comparatively, for pure $\mathrm{Ni}$, the 
permeability $\left(\mu_{r}=60\right)$ and saturation magnetization $\left(\mu_{0} M_{s}=0.616 \mathrm{~T}\right)$ are lower, and the anisotropic constant $\left(100 \mathrm{~J} / \mathrm{m}^{3}\right)$ is smaller. The magnetic properties of the FeCuNbSiB ribbons and the Ni plate are significantly different, which results in an additional magnetic field being generated in the Ni plate due to the flux concentration effect of the high-permeability FeCuNbSiB ribbons [9,23]. According to previous reports $[9,23]$, a magnetic material with high-permeability can be assumed as a static magnetic source, which results in the flux concentration effect and generates an effective magnetic field around it. Thus, the effective magnetic field in $\mathrm{Ni}$ of $\mathrm{FeCuNbSiB} / \mathrm{Ni}\left(H_{e f f, m 2 f}\right)$ is larger than that of a single Ni plate without $\mathrm{FeCuNbSiB}\left(H_{\text {eff, } m 2 n}\right)$ due to the flux concentration effect of FeCuNbSiB. Here, we assume that the coefficient of the flux concentration effect is $\delta$. So, both $H_{\mathrm{dc}}$ and $H_{\mathrm{ac}}$ in $\mathrm{Ni}$ are amplified $\delta$ times. In general, $H_{\mathrm{dc}}$ is far larger than the $H_{\mathrm{ac}}$. Furthermore, the internal magnetic field in the Ni plate is influenced by the demagnetizing field $H_{d}$ which is completely opposite to the magnetization. The effective magnetic field $H_{\mathrm{eff}, m 2 n}$ of the single Ni plate and $H_{\mathrm{eff}, m 2 f}$ of Ni in the $\mathrm{FeCuNbSiB} / \mathrm{Ni}$ laminate are

$$
\begin{aligned}
& H_{e f f, m 2 n}=\frac{H_{\mathrm{dc}}+H_{\mathrm{ac}}}{1+N_{d, m 2}\left(\mu_{r, m 2}-1\right)} \\
& H_{e f f, m 2 \mathrm{f}}=\frac{\delta\left(H_{\mathrm{dc}}+H_{\mathrm{ac}}\right)}{1+N_{d, m 2}\left(\mu_{r, m 2}-1\right)}
\end{aligned}
$$

From Equation (9a) and (9b), the piezomagnetic coefficient of single $\mathrm{Ni} d_{33, m 2 n}$, and $\mathrm{Ni}$ in $\mathrm{FeCuNbSiB} / \mathrm{Ni} d_{33, m 2 f}$ are

$$
\begin{aligned}
& d_{33 e f f, m 2 n}=2 \lambda_{s, m 2}\left(\operatorname{coth}\left(\eta_{m 2} H_{e f f, m 2 n}\right)-\frac{1}{\eta_{m 2} H_{e f f, m 2 n}}\right) \times\left[\eta_{m 2}\left(1-\operatorname{coth}\left(\eta_{m 2} H_{e f f, m 2 n}\right)^{2}\right)+\frac{1}{\eta_{m 2}\left(H_{e f f, m 2 n}\right)^{2}}\right] \\
& d_{33 e f f, m 2 f}=2 \delta \lambda_{s, m f}\left(\operatorname{coth}\left(\eta_{m 2} H_{e f f, m 2 f}\right)-\frac{1}{\eta_{m 2} H_{e f f, m 2 f}}\right) \times\left[\eta_{m 2}\left(1-\operatorname{coth}\left(\eta_{m 2} H_{e f f, m 2 f}\right)^{2}\right)+\frac{1}{\eta_{m 2}\left(H_{e f f, m 2 f}\right)^{2}}\right]
\end{aligned}
$$

According to the principle of magnetic charge, the magnetic charges of the Ni plate are gathered and distributed at the two end faces. So the amplitude of the magnetic field at the face ends of the Ni plate can reflect its magnetization state, assuming that the amplitude of the magnetic field at the end of the single $\mathrm{Ni}$ plate is $H_{\mathrm{Ni}}$ and $\mathrm{Ni}$ in $\mathrm{FeCuNbSiB} / \mathrm{Ni}$ is $H_{\mathrm{F}-\mathrm{Ni}}$. In order to obtain the values of $H_{\mathrm{Ni}}$ and $H_{\mathrm{F}-\mathrm{Ni}}$, we use the Ansoft 11.0 software package (2D magnetostatic simulation) so we can obtain $\delta=H_{\mathrm{F}-\mathrm{Ni}} / H_{\mathrm{Ni}}$. The two-dimensional simulation parameters are set as follows: permanent magnet NdFeB-N35 (75 mm length $\times 5 \mathrm{~mm}$ width, the distance of two magnets is $90 \mathrm{~mm}) ; \mathrm{Ni}(\mu=220,12 \mathrm{~mm}$ length $\times 1 \mathrm{~mm}$ height); and $\mathrm{FeCuNbSiB}\left(\mu=3 \times 10_{4}, 75 \mathrm{~mm}\right.$ length $\times 0.03 \mathrm{~mm}$ height $)$. When the layers of $\mathrm{FeCuNbSiB}$ ribbon $L=1$, the distribution of flux lines of $\mathrm{FeCuNbSiB} / \mathrm{Ni}$; the distribution of the magnetic field of the $\mathrm{FeCuNbSiB} / \mathrm{Ni}$ laminate are shown in Figure 4.

As shown in Figure 5, when centerline $x$ changes, the magnetic field $H$ curves along the $x$-direction near the two ends of the Ni plate. Clearly, the magnetic field of the Ni plate in FeCuNbSiB/Ni is far greater than that in the single Ni plate, which is attributed to the flux concentration effect of FeCuNbSiB. For a single Ni plate, $H_{\mathrm{Ni}}$ is $908(\mathrm{~A} / \mathrm{m})$ at the end face. When the FeCuNbSiB layer number $L=1-5, H_{\mathrm{F}-\mathrm{Ni}}$ at the end faces of the Ni plate in $\mathrm{FeCuNbSiB} / \mathrm{Ni}$ are $1631.15395(\mathrm{~A} / \mathrm{m}), 1653.96385(\mathrm{~A} / \mathrm{m})$, $1658.66092(\mathrm{~A} / \mathrm{m}), 1662.78705(\mathrm{~A} / \mathrm{m})$, and $1657.69218(\mathrm{~A} / \mathrm{m})$, respectively. When $L=4$, the end face magnetic field intensity is the maximum. Therefore, according to the simulation results, the magnetic field convergence factor is $\delta=H_{\mathrm{F}-\mathrm{Ni}} / \mathrm{H}_{\mathrm{Ni}}$, which is about 1.83 times. 
(b) $\mathrm{H}[\mathrm{A} / \mathrm{m}]$

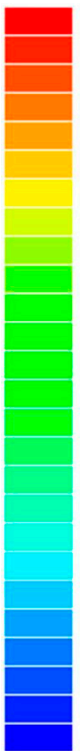

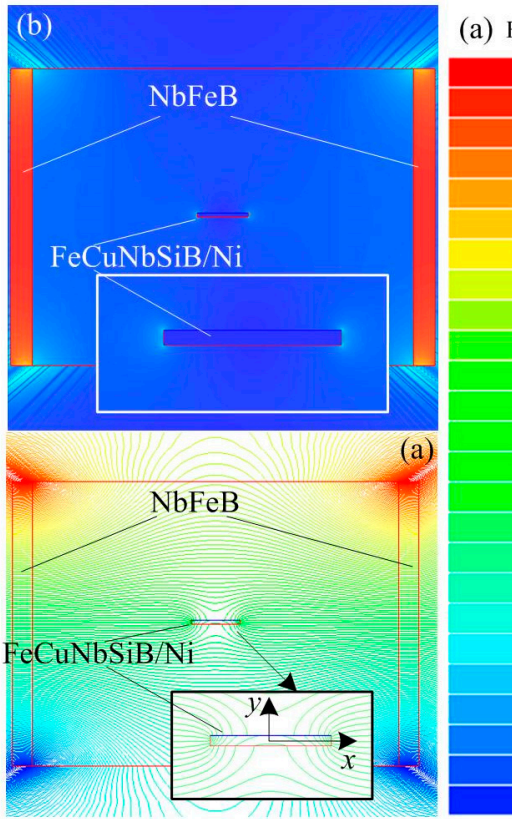

(a) Flux Lines $(\mathrm{Wb} / \mathrm{m})$
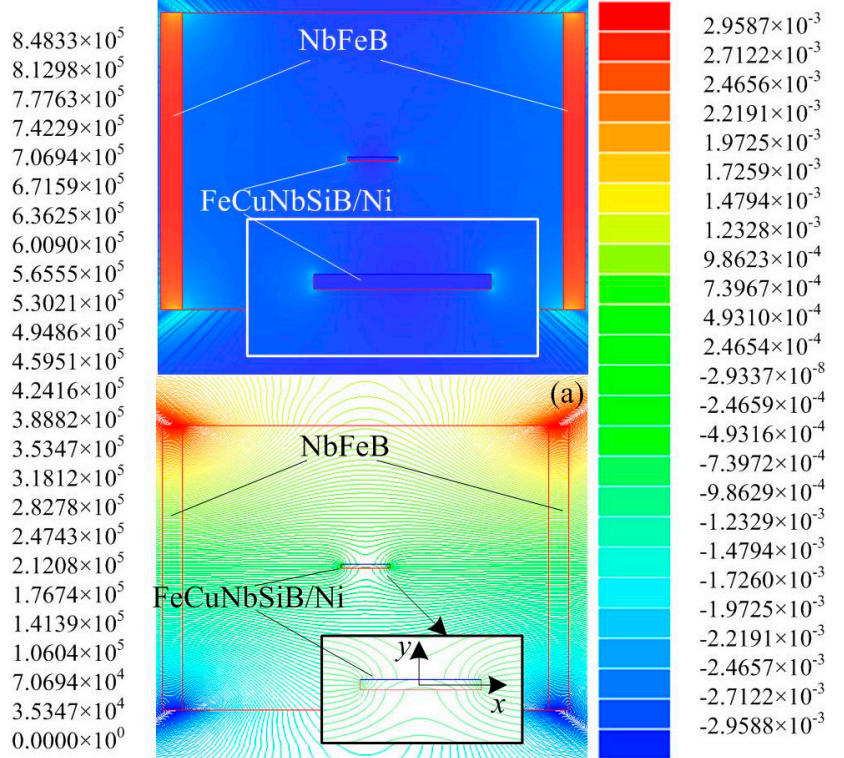

Figure 4. When the layers of the FeCuNbSiB ribbon $L=1$, the distribution diagrams of (a) flux lines and (b) magnetic field of the $\mathrm{FeCuNbSiB} / \mathrm{Ni}$ laminate.

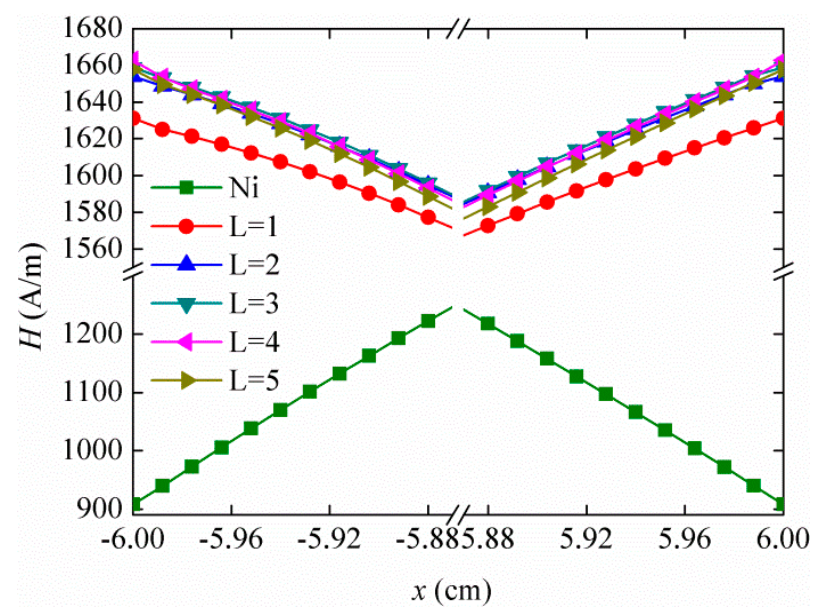

Figure 5. The amplitude of the magnetic field along $x$-direction for the single Ni plate and $\mathrm{Ni}$ in $\mathrm{FeCuNbSiB} / \mathrm{Ni}(L=1-5)$.

\section{Results and Discussion}

In the present work, we compare the calculated results to the experiments to prove the effectiveness of our model for the magneto-mechanical and ME coupling characteristics. Substituting the material's parameters shown in Table 1 into Equations (5), (8), (10a) and (10b), the theoretical results of $d_{33, m}$ versus $H_{\mathrm{dc}}$ for $\mathrm{FeCuNbSiB} / \mathrm{Ni}$ can be obtained, as shown in Figure 6. The FeCuNbSiB is a positive magnetostrictive material, and $\mathrm{Ni}$ is a negative magnetostrictive material. In the calculation, $d_{33, m 1}$ and $d_{33, m 2}$ in Equation (5) are converse.

In experiments, the Doppler vibrometer (Polytec OFV-5000) was used to measure the vibration velocity $v$ at the end faces of the $\mathrm{FeCuNbSiB} / \mathrm{Ni}$ laminate, then the $d_{33, m}$ was calculated by $d_{33, m}=d \lambda / d H=v /\left(\pi f l H_{\mathrm{ac}}\right)$. The $H_{\mathrm{ac}}$ is the external AC magnetic field, $f$ is the vibration frequency, and $l$ is the length. It shows clearly that the calculated $d_{33, m}$ agrees well with the experimental data, and a large zero-biased $d_{33, m}$ of $\mathrm{FeCuNbSiB} / \mathrm{Ni}$ can be achieved. The errors in experimental data originally 
from the magnetization state of piezomagnetic material is inconsistent in each measurement during all the processes of repeated measurements.

From Figure 6, there is an error between the calculation result and the experimental values. The reason for this phenomenon is that the experimental device was not fully demagnetized in the actual experimental process, and some simulation parameters are used in the theoretical calculation. We can obtain the results:

(i) For the outstanding soft magnetic performance of $\mathrm{FeCuNbSiB}$, the magneto-mechanical coupling of FeCuNbSiB is larger than $\mathrm{Ni}$ in Section $1\left(d_{33, m 1}>d_{33, m 2}\right)$. As $H_{\mathrm{dc}}$ increases, the $d_{33, m 1}$ increases rapidly, and the maximum value $P_{\mathrm{I}}=12.9 \mathrm{~nm} / \mathrm{A}$ is obtained under the optimal $H_{\mathrm{dc}}=1184 \mathrm{~A} / \mathrm{m}$.

(ii) When $H_{\mathrm{dc}}$ increases to a certain value, the magneto-mechanical effects of FeCuNbSiB and $\mathrm{Ni}$ cancel each other out, and the dynamic $d_{33, m}$ appears at the zero-cross point as shown in Figure 6.

(iii) Due to the flux concentrate effect of $\mathrm{FeCuNbSiB}$, the internal magnetic moments of Ni rotate rapidly. Then, the $d_{33, m 2}$ becomes larger. In Section 2 of Figure 6 , the $d_{33, m}$ is originated from the magneto-mechanical coupling of Ni with the flux concentrate effect. After the zero-crossing point, the $d_{33, m}$ rapidly increases to the maximum value $P_{\mathrm{II}}$. However, when $H_{\mathrm{dc}}$ increases to a certain value, FeCuNbSiB gradually saturates, the flux concentration effect is abate, which results in the appearance of the downward trend in the II area.

(iv) With the increase of $H_{\mathrm{dc}}$, the flux concentration effect gradually decreases, so in Section 3 of Figure 6, the $d_{33, m}$ is attributed to the magneto-mechanical coupling of Ni itself. From Equation (10a) and (10b), we assume that $d_{33, m 2 f}=d_{33, m 2 n}$ when $H_{\mathrm{dc}}=H_{n}$, and $d_{33, m 2 f}$ is far larger than $d_{33, m 2 n}$ when $H_{\mathrm{dc}}<H_{n}$. As $H_{\mathrm{dc}}$ increases gradually, the flux concentration effect disappears when $H_{\mathrm{dc}}>H_{n}$. So for the $d_{33, m}$ of the Ni plate in the theoretical calculation, $d_{33, \mathrm{~m} 2}=d_{33, m 2 f}$ when $H_{\mathrm{dc}}<$ $H_{n}$ and $d_{33, \mathrm{~m} 2}=d_{33, m 2 n}$ when $H_{\mathrm{dc}}>H_{n}$. From Figure 6, the magnetic field $H_{n}$ for $d_{33, m 2 f}=d_{33, m 2 n}$ in Equation (10a) and (10b) is $\sim 12,800(\mathrm{~A} / \mathrm{m})$ in experiments, and is $\sim 15,600(\mathrm{~A} / \mathrm{m})$ in theory.

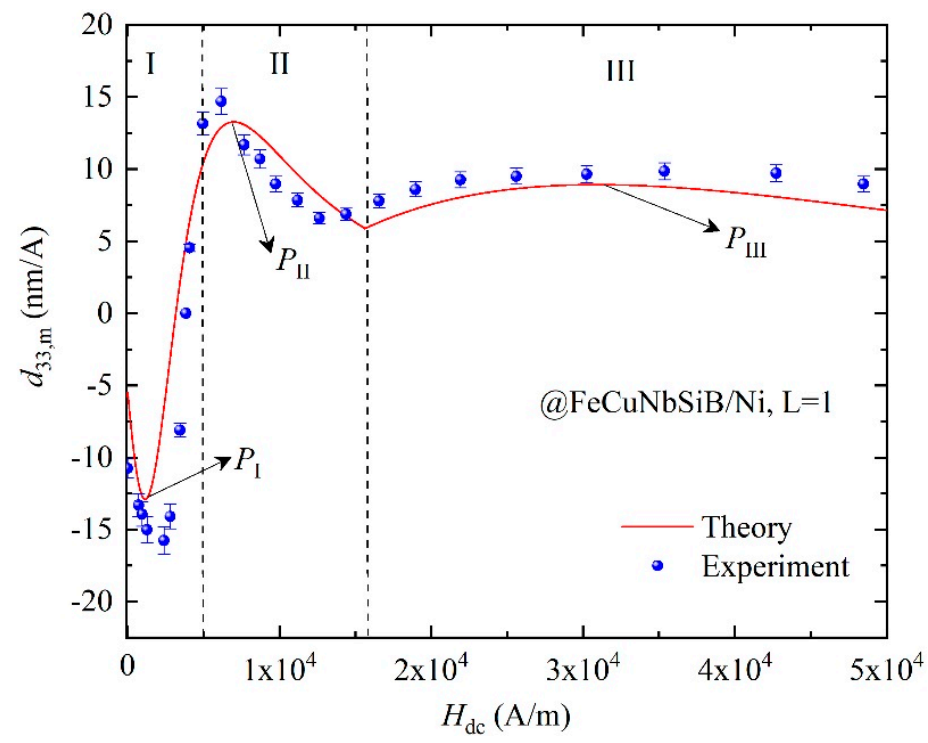

Figure 6. The calculated and experimental $d_{33, m}$ as a function of $H_{\mathrm{dc}}$ for the FeCuNbSiB/Ni laminate. The layer of FeCuNbSiB is one $(L=1)$.

Using the method of the magneto-mechanical-electric equivalent circuit, the longitudinal resonant ME voltage coefficient $\alpha_{M E, \mathrm{r}}$ for L-T mode laminate structure is given by [24,25]

$$
\alpha_{M E, \mathrm{r}}=\left|\frac{d V}{t d H_{3}}\right|=\frac{8 Q_{m}}{\pi^{2}} \frac{n(1-n) d_{33, m} d_{31, p}}{\varepsilon_{33}\left[\mathrm{f}\left(1-k_{31}^{2}\right) s_{11}^{E}+(1-n) s_{33}^{H}\right]}
$$


where $Q_{m}$ is the mechanical quality factor, $n$ is the volume ratio of the magnetostrictive phase, $\varepsilon_{33}$ is the permittivity tensor, $d_{33, m}$ is the piezomagnetic coefficient, $d_{31, p}$ is the piezoelectric coefficient, $k_{31}$ is the electromechanical coupling coefficient of the piezoelectric material, $s_{11}^{E}$ is the elastic compliance of the piezoelectric phase, and $s_{33}^{H}$ is the elastic compliance of the piezomagnetic phase.

Using $\mathrm{Pb}\left(\mathrm{Zr}_{1-\mathrm{x}} \mathrm{Ti}_{\mathrm{x}}\right) \mathrm{O}_{3}(\mathrm{PZT})$ ceramic as the piezoelectric material, the ME composite FeCuNbSiB/Ni/PZT can be obtained. Taking the material's parameters in Table 1 and Equation (5) into Equation (11), the theoretical $\alpha_{M E, \mathrm{r}}$ can be obtained, as shown in Figure 7. For comparison, the experimental data for $\mathrm{FeCuNbSiB} / \mathrm{Ni} / \mathrm{PZT}$ with $L=1$ are used, as shown in Figure 7 . It can be seen from the figure that the theoretical calculation and experimental value of $\alpha_{M E, \mathrm{r}}$ is consistent with the variation trend of the $H_{\mathrm{dc}}$. From Equation (11), one can derive $\alpha_{M E, \mathrm{r}} \propto d_{33, m}$. Therefore, the curve of $\alpha_{M E, \mathrm{r}}$ vs. $H_{\mathrm{dc}}$ in Figure 7 is consistent with that of $d_{33, m}$ vs. $H_{\mathrm{dc}}$ in Figure 6. The theoretical calculation result is different from the experimental result. The reason is that the experimental device is not demagnetized in the actual experimental process. In addition, the composite structure of $\mathrm{FeCuNbSiB} / \mathrm{Ni} / \mathrm{PZT}$ has a certain thickness of glue, which was not considered in the theoretical calculation results.

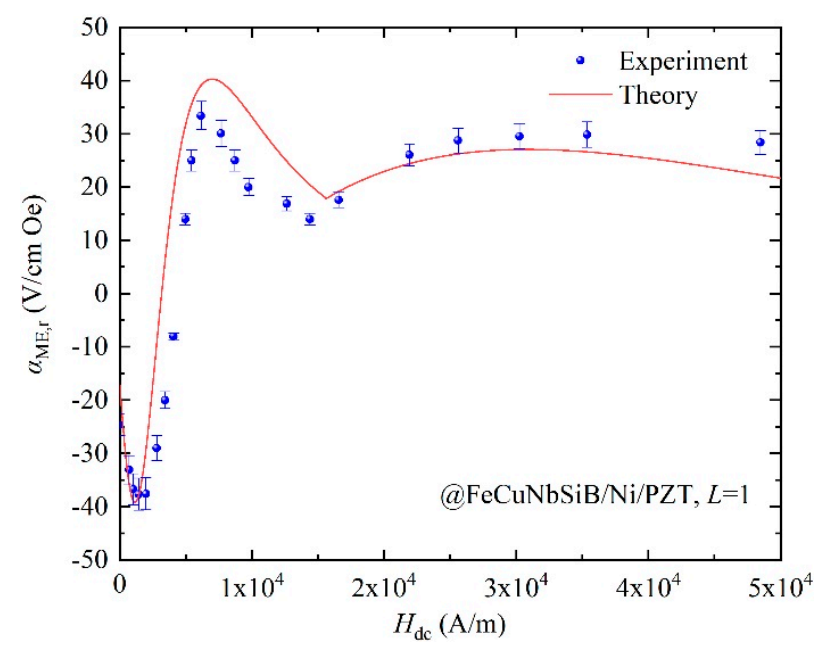

Figure 7. The theoretical and experimental $\alpha_{M E, \mathrm{r}}$ vs. $H_{\mathrm{dc}}$ for the FeCuNbSiB/Ni/PZT composite.

Table 1. Material parameters of the FeCuNbSiB ribbon, Ni plate, FeNi plate, and the $\mathrm{Pb}\left(\mathrm{Zr}_{1-\mathrm{x}} \mathrm{Ti}_{\mathrm{x}}\right) \mathrm{O}_{3}$ (PZT) plate.

\begin{tabular}{ccccccccc}
\hline Material & $\chi_{m}$ & $\begin{array}{c}\mu_{0} \boldsymbol{M}_{\mathbf{s}} \\
(\mathbf{T})\end{array}$ & $\begin{array}{c}\lambda_{s} \\
(\mathbf{p p m})\end{array}$ & $\begin{array}{c}L \times w \times t \\
\left(\mathbf{m m}^{\mathbf{3}}\right)\end{array}$ & $\begin{array}{c}s_{33} \\
\left(\mathbf{1 0 ^ { - 1 2 }} \mathbf{m}^{2} / \mathbf{N}\right)\end{array}$ & $\varepsilon_{33} / \varepsilon_{\mathbf{0}}$ & $\begin{array}{c}\rho \\
\left(\mathrm{g} / \mathbf{c m}^{3}\right)\end{array}$ & $Q_{\text {mech }}$ \\
\hline $\mathrm{Ni}$ & 200 & 0.616 & 40 & $12 \times 6 \times 1$ & 4.9 & & 8.9 & 150 \\
\hline $\mathrm{FeCuNbSiB}$ & $4 \times 10^{5}$ & 1.25 & 2.7 & $12 \times 6 \times 0.03$ & 5.2 & & 7.25 & 1000 \\
\hline $\mathrm{FeNi}$ & 700 & 0.75 & 20 & $12 \times 6 \times 0.6$ & 5 & 8 & 200 \\
\hline $\mathrm{PZT}$ & & & & $12 \times 6 \times 1$ & 15.3 & 1750 & 7.75 & 1000 \\
\hline
\end{tabular}

In previous work, FeCuNbSiB shows positive magnetostriction and $\mathrm{Ni}$ shows negative magnetostriction. In order to check the validity of the theoretical model, the other magnetostrictive material FeNi with positive magnetostrictive properties was chosen to replace Ni. Then, we also compared the theoretical results with the experimental data using MGFM FeCuNbSiB/FeNi and ME laminate $\mathrm{FeCuNbSiB} / \mathrm{FeNi} / \mathrm{PZT}$.

Substituting the material's parameters, shown in Table 1, into Equations (5), (8), (10a) and (10b), the calculated $d_{33, m}$ versus $H_{\mathrm{dc}}$ for $\mathrm{FeCuNbSiB} / \mathrm{FeNi}$ is achieved, as shown in Figure 8 . The result in Figure 8 is different from that of Figure 6. The reason is that the Ni shows negative magnetostriction and FeNi shows positive magnetostriction. In Section 1, the $d_{33, \mathrm{~m}}$ of FeCuNbSiB/FeNi originates from $d_{33, m 1}$ of $\mathrm{FeCuNbSiB}$ and $d_{33, m f}$ (Equation (10b)) of FeNi with the flux concentration effect. In Section 2, the $d_{33, m}$ of $\mathrm{FeCuNbSiB} / \mathrm{FeNi}$ originates from $d_{33, m n}$ (Equation (10a)) of FeNi. 
To make further efforts to verify the proposed theoretical model, the FeCuNbSiB/FeNi/PZT composite is used, and the calculated and experimental data are shown in Figure 9. The curve of $\alpha_{M E, \mathrm{r}}$ vs. $H_{\mathrm{dc}}$ in Figure 9 is consistent with that of $d_{33, m}$ vs. $H_{\mathrm{dc}}$ in Figure 8 . The theoretical result for $d_{33, m}$, and $\alpha_{M E, \mathrm{r}}$ agrees well with those of experiments. Clearly, the proposed theoretical models are also proven to be useful for the other MGFM and ME laminated composite with MGFM.

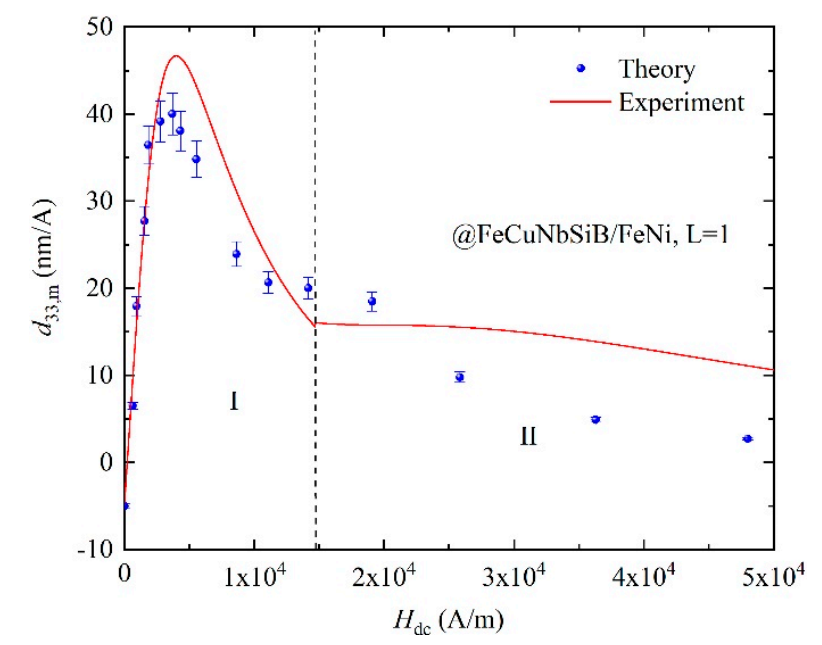

Figure 8. Dynamic piezomagnetic coefficient $d_{33, m}$ of $\mathrm{FeCuNbSiB} / \mathrm{FeNi}$ as a function of $H_{\mathrm{dc}}$. The layer of $\mathrm{FeCuNbSiB}$ is one $(L=1)$.

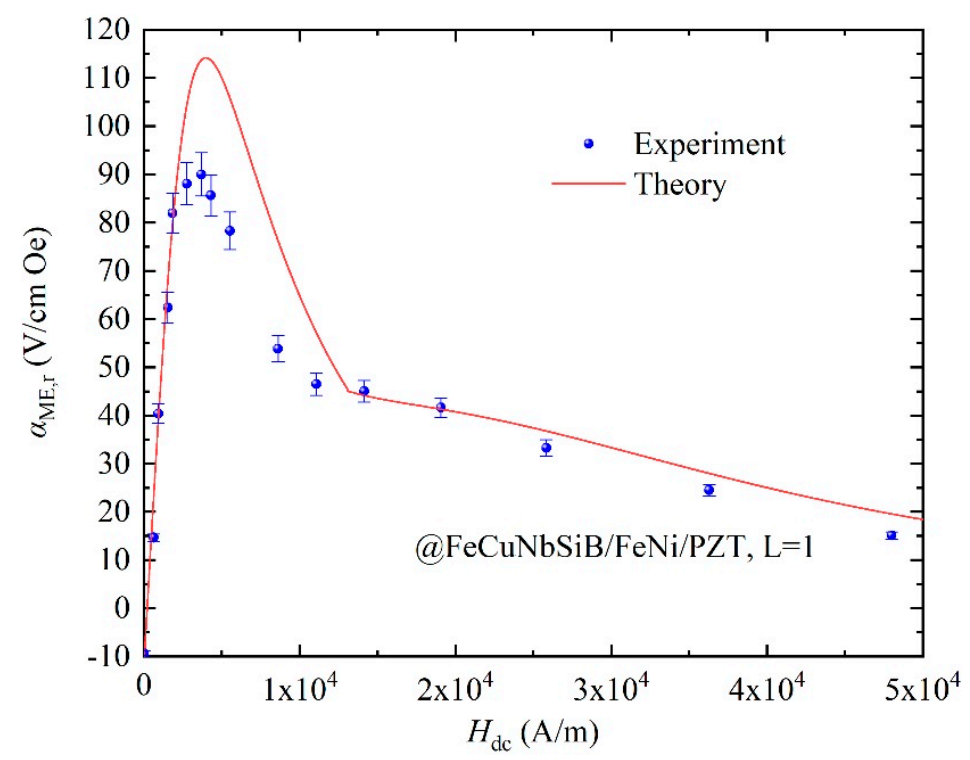

Figure 9. The theoretical and experimental $\alpha_{M E, \mathrm{r}}$ as a function of $H_{\mathrm{dc}}$ for $\mathrm{FeCuNbSiB} / \mathrm{FeNi} / \mathrm{PZT}$.

\section{Conclusions}

According to the nonlinear constitutive model of piezomagnetic material, magnetoelectric equivalent circuit method, and simulation software Ansoft, a theoretical model about $d_{33, m}$ vs. $H_{\mathrm{dc}}$ is effectively and accurately established for MGFM. The theoretical $d_{33, m}$ of FeCuNbSiB/Ni, or $\mathrm{FeCuNbSiB} / \mathrm{FeNi}$ has been compared with the experimental data. By using the presented piezomagnetic model of MGFM, the resonant ME voltage coefficient $\alpha_{M E, r}$ as a function of $H_{\mathrm{dc}}$ for $\mathrm{FeCuNbSiB} / \mathrm{Ni} / \mathrm{PZT}$ or $\mathrm{FeCuNbSiB} / \mathrm{FeNi} / \mathrm{PZT}$ composite has been investigated. The theoretical calculated result agrees well with that of the experiments. Therefore, the proposed piezomagnetic model will provide an effective tool to fully understand the dynamic magneto-mechanical behavior of MGFM and self-biased ME 
coupling of composites with MGFM. It is useful to guide the designing of ME devices with MGFM, such as magnetic sensors and energy harvesters.

Author Contributions: Conceptualization, H.Z. and C.L.; methodology, C.L.; software, F.Y.; validation, H.Z. and X.J.; investigation, H.Z.; resources, C.L. and H.G.; data curation, H.Z.; writing-original draft preparation, H.Z.; writing-review and editing, C.L. and Z.C.; supervision, C.L. and X.C.; funding acquisition, C.L. and A.Y. All authors have read and agreed to the published version of the manuscript.

Funding: This work was supported by the National Natural Science Foundation of China (Grant Nos. 61801402, 51775452), Science and Technology program of Sichuan Province (20JDJQ0038), Fundamental Research Funds for the Central Universities (2682020CX26), and the Science and Technology Program of State Grid Jiangxi Electric Power Co., Ltd. (No. 521820180004).

Conflicts of Interest: The authors declare no conflict of interest.

\section{References}

1. Chu, Z.; PourhosseiniAsl, M.; Dong, S. Review of multi-layered magnetoelectric composite materials and devices applications. J. Phys. D Appl. Phys. 2018, 51, 243001. [CrossRef]

2. Ou-Yang, J.; Liu, X.; Zhou, H.; Zou, Z.; Yang, Y.; Li, J.; Zhang, Y.; Zhu, B.; Chen, S.; Yang, X. Magnetoelectric laminate composites: An overview of methods for improving the DC and low-frequency response. J. Phys. D Appl. Phys. 2018, 51, 324005. [CrossRef]

3. Carlos, A.F.V.; Hoffman, J.; Ahn, C.H.; Ramesh, R. Magnetoelectric coupling effects in multiferroic complex oxide composite Structures. Adv. Mater. 2010, 22, 2900-2918.

4. Lu, C.; Li, P.; Wen, Y.; Yang, A. Large Self-Biased Magnetoelectric Properties in Heterostructure of Graded-Magnetostrictive Layers and a Rosen-Type Piezoelectric Transformer. IEEE Sens. J. 2014, 15, 402-407. [CrossRef]

5. Wang, W.; Ou-Yang, J.; Wu, X.; Zhang, Y.; Yang, X.; Chen, S. High-Isotropic Magnetic Sensor Based on Ring Terfenol-D/PZT Composites. IEEE Trans. Magn. 2018, 54, 1-5. [CrossRef]

6. Jing, W.Q.; Fang, F. Stress-induced self-biasing of magnetoelectric coupling in embedded Ni/PZT/FeNi composite. Appl. Phys. Lett. 2015, 106, 212901. [CrossRef]

7. Nguyen, T.; Mininger, X.; Daniel, L.; Bouillault, F. Influence of Mechanical Boundary Conditions on Magnetoelectric Sensors. IEEE Trans. Magn. 2013, 49, 2009-2012. [CrossRef]

8. Lu, C.; Zhou, H.; Yang, A.; Ou, Z.; Yu, F.; Gao, H. Nonlinear Magnetoelectric Response of Fe73.5Cu1Nb3Si13.5B9/Piezofiber Composite for a Pulsed Magnetic Field Sensor. Materials 2019, 12, 2866. [CrossRef]

9. Li, M.; Matyushov, A.; Dong, C.; Chen, H.; Lin, H.; Nan, T.; Qian, Z.; Rinaldi, M.; Lin, Y.; Sun, N.-X. Ultra-sensitive NEMS magnetoelectric sensor for picotesla DC magnetic field detection. Appl. Phys. Lett. 2017, 110, 143510. [CrossRef]

10. Sadovnikov, A.V.; Grachev, A.A.; Sheshukova, S.E.; Sharaevskii, Y.P.; Serdobintsev, A.A.; Mitin, D.M.; Nikitov, S.A. Magnon Straintronics: Reconfigurable Spin-Wave Routing in Strain-Controlled Bilateral Magnetic Stripes. Phys. Rev. Lett. 2018, 120, 257203. [CrossRef]

11. Sadovnikov, A.V.; Grachev, A.A.; Serdobintsev, A.A.; Sheshukova, S.E.; Yankin, S.S.; Nikitov, S.A. Magnon Straintronics to Control Spin-Wave Computation: Strain Reconfigurable Magnonic-Crystal Directional Coupler. IEEE Magn. Lett. 2019, 10, 1-5. [CrossRef]

12. Gusev, N.S.; Sadovnikov, A.V.; Nikitov, S.A.; Sapozhnikov, M.V.; Udalov, O.G. Manipulation of the Dzyaloshinskii-Moriya Interaction in Co/Pt Multilayers with Strain. Phys. Rev. Lett. 2020, 124, 157202. [CrossRef]

13. Leung, C.M.; Li, J.; Viehland, D.; Zhuang, X. A review on applications of magnetoelectric composites: From heterostructural uncooled magnetic sensors, energy harvesters to highly efficient power converters. J. Phys. D Appl. Phys. 2018, 51, 263002. [CrossRef]

14. Zhou, Y.; Priya, S. Near-flat self-biased magnetoelectric response in geometry gradient composite. J. Appl. Phys. 2014, 115, 104107. [CrossRef]

15. Zhang, J.; Li, P.; Wen, Y.; He, W.; Yang, A.; Lu, C. Giant self-biased magnetoelectric response with obvious hysteresis in layered homogeneous composites of negative magnetostrictive material Samfenol and piezoelectric ceramics. Appl. Phys. Lett. 2013, 103, 202902. [CrossRef] 
16. Tang, C.; Lu, C. Strong self-biased magnetoelectric charge coupling in a homogenous laminate stack for magnetic sensor. J. Alloy Compd. 2016, 686, 723-726. [CrossRef]

17. Lage, E.; Kirchhof, C.; Hrkac, V.; Kienle, L.; Jahns, R.; Knöchel, R.; Quandt, E.; Meyners, D. Exchange biasing of magnetoelectric composites. Nat. Mater. 2012, 11, 523-529. [CrossRef]

18. Röbisch, V.; Yarar, E.; Urs, N.O.; Teliban, I.; Knöchel, R.; Mccord, J.; Quandt, E.; Meyners, D. Exchange biased magnetoelectric composites for magnetic field sensor application by frequency conversion. J. Appl. Phys. 2015, 117, 17B513. [CrossRef]

19. Laletin, U.; Sreenivasulu, G.; Petrov, V.M.; Garg, T.; Kulkarni, A.R.; Venkataramani, N.; Srinivasan, G. Hysteresis and remanence in magnetoelectric effects in functionally graded magnetostrictive-piezoelectric layered composites. Phys. Rev. B 2012, 85, 104404. [CrossRef]

20. Ou, Z.; Lu, C.; Yang, A.; Zhou, H.; Cao, Z.; Zhua, R.; Gao, H. Self-biased magnetoelectric current sensor based on SrFe12O19/FeCuNbSiB/PZT composite. Sens. Actuators A 2019, 290, 8-13. [CrossRef]

21. Mandal, S.K.; Sreenivasulu, G.; Petrov, V.M.; Srinivasan, G. Magnetization-graded multiferroic composite and magnetoelectric effects at zero bias. Phys. Rev. B 2011, 84, 014432. [CrossRef]

22. Chen, L.; Li, P.; Wen, Y.; Zhu, Y. Theoretical analyses of nonlinear magnetoelectric response in self-biased magnetostrictive/piezoelectric laminated composites. Compos. Struct. 2015, 119, 685-692. [CrossRef]

23. Lu, C.; Li, P.; Wen, Y.; Yang, A.; He, W.; Zhang, J.; Yang, J.; Wen, J.; Zhu, Y.; Yu, M. Investigation of magnetostrictive/piezoelectric multilayer composite with a giant zero-biased magnetoelectric effect. Appl. Phys. A 2013, 113, 413-421. [CrossRef]

24. Dong, S.; Li, J.-F.; Viehland, D. Longitudinal and transverse magnetoelectric voltage coefficients of magnetostrictive/piezoelectric laminate composite: Theory. IEEE Trans. Ultrason. Ferroelectr Freq. Control 2003, 50, 1253-1261. [CrossRef]

25. Yang, F.; Wen, Y.-M.; Li, P.; Zheng, M.; Bian, L. Resonant magnetoelectric response of magnetostrictive/piezoelectric laminate composite in consideration of losses. Sens. Actuators A Phys. 2008, 141, 129-135. [CrossRef]

26. Zheng, X.; Liu, X.E. A nonlinear constitutive model for Terfenol-D rods. J. Appl. Phys. 2005, 97, 053901. [CrossRef]

(C) 2020 by the authors. Licensee MDPI, Basel, Switzerland. This article is an open access article distributed under the terms and conditions of the Creative Commons Attribution (CC BY) license (http://creativecommons.org/licenses/by/4.0/). 